ISSN: $1130-3743$ - e-ISSN: 2386-5660

DOI: http://dx.doi.org/10.14201/teoredu301217240

\title{
PROFUNDIZANDO EN LA TRANSICIÓN ENTRE EDUCACIÓN INFANTIL Y EDUCACIÓN PRIMARIA: LA PERSPECTIVA DE FAMILIAS Y PROFESORADO
}

\author{
Deepening the transition between childhood education and \\ primary education: the perspective of families and teachers
}

\section{Approfondir la transition entre l'école maternelle et l'école élémentaire: le point de vue des familles et des enseignants}

Ana Castro Zubizarreta, Pilar EzQuerra Muñoz y Javier Argos GonzÁlez Universidad de Cantabria. Facultad de Educación. Departamento de Educación. Avda.de los Castros, s/n.39005 Santander. castroza@unican.es; ezquermp@unican.es; argosj@unican.es

Fecha de recepción: enero de 2018

Fecha de aceptación: marzo de 2018

RESUMEN

El trabajo pretende conocer la perspectiva mantenida por familias y profesorado en relación con diferentes elementos del proceso de transición entre Educación Infantil y Educación Primaria: su importancia o trascendencia, los cambios que se producen y los desafíos a los que se enfrentan los pequeños, así como los mecanismos y actuaciones implementados por los centros para favorecerlo. 460 familias y profesores participaron en el estudio cumplimentando un cuestionario. Los resultados ponen de manifiesto la existencia de una brecha importante de pensamiento entre familias y profesorado en torno a la importancia y la trascendencia concedida a las transiciones que se viven en la primera infancia, a pesar de encontrar también otros aspectos convergentes. Teniendo en cuenta dichos hallazgos se anima a la generación de programas de transición educativa adaptados a las necesidades y 
demandas de cada centro escolar, que favorezcan la participación de todas las personas implicadas en este importante proceso.

Palabras clave: transición educativa; Educación Infantil; Educación Primaria; familias; profesorado.

\section{SUMMARY}

The work aims to know the perspective maintained by families and teachers in relation to different elements of the transition process between Childhood Education and Primary Education: its importance or transcendence, the changes that occur and the challenges faced by the children, as well as the mechanisms and actions implemented by the centers to encourage it. 460 families and professors participated in the study completing a questionnaire. The results show the existence of a significant gap in thinking about the importance and transcendence given to the transitions that are experienced in early childhood between families and teachers despite also finding other aspects converging. Considering these findings, the generation of educational transition programs adapted to the needs and demands of each school which encourage the participation of all the people involved in this important process.

Key words: educational transition; Early Childhood Education; Primary Education; families; teachers.

\section{SOMMAIRE}

L'objectif de ce travail est de connaître le point de vue qu'ont les familles et les enseignants sur les différents éléments du processus de transition éducative entre l'école maternelle et l'école élémentaire: son importance ou transcendance, les changements qui se produisent et les défis rencontrés par les enfants, ainsi que les mécanismes et actions développé par les centres pour le favoriser. 460 familles et enseignants ont participé à l'étude en remplissant un questionnaire. Les résultats confirment l'existence d'un important fossé de pensée entre les familles et les enseignants par rapport à l'importance et à la transcendance accordées aux transitions qui sont vécues, bien qu'il existe également d'autres aspects convergents. Sur la base de ces résultats, nous encourageons la création de programmes de transition éducative adaptés aux besoins et aux exigences de chaque école, qui favorisent la participation de toutes les personnes impliquées dans ce processus important.

Mots clés: transition éducative; École Maternelle; École Élémentaire; familles; enseignants. 
A. CASTRO ZUBIZARRETA, P. EZQUERRA MUÑOZ Y J. ARGOS GONZÁLEZ

\section{LAS TRANSICIONES EDUCATIVAS. CUESTIONES INTRODUCTORIAS}

Abordar el estudio de la transición entre etapas educativas supone atender a diversos actores y escenarios inmersos en este importante proceso vital que experimentan los niños. Vloger, Crivello y Woodhead (2008, 2) definen las transiciones como «los acontecimientos y/o procesos clave que ocurren en períodos o coyunturas específicos a lo largo del curso de la vida». Las transiciones educativas hacen, por tanto, referencia al cambio que efectúan los niños desde una fase educativa a otra. Concretamente, los referidos investigadores describen cómo a lo largo del proceso de transición educativa se van produciendo en los niños unos ajustes en la forma de percibir y adaptase al medio, de relacionarse con ellos mismos, con los otros y con el mundo, que serán facilitados o limitados por la forma en la que se dispongan y organicen los contextos.

Autores como Abello (2008), Castro, Ezquerra y Argos (2012) o Gairín (2005) coinciden en señalar que la transición educativa puede ser entendida como una oportunidad o como un problema. Al respecto, Featherstone (2004), Sayers et al. (2012) y Schulting, Malone y Dodge (2005) señalan que la vivencia de una transición educativa exitosa por parte de los niños repercute de forma positiva en el éxito académico y social de estos. La transición educativa se presenta, por tanto, como una oportunidad de aprendizaje académico y personal.

Por su parte, Abello (2008) y Rosier y McDonald (2011), desde la perspectiva de contemplar la transición como problema, señalan que esta puede ser la causa del miedo frente a lo nuevo que genera confusión y ansiedad, sensaciones que pueden condicionar el comportamiento ulterior del niño. Asimismo, Conyer, Reynolds y Ou (2003) sugieren que los niños pueden estar en mayor riesgo de fracaso escolar y de tener problemas de ajuste social cuando experimentan una transición ineficaz o dificultosa entre etapas educativas. De las anteriores consideraciones, podríamos concebir la transición a la Educación Primaria como uno de los grandes desafíos a superar por los niños en los primeros años de su infancia (Dockett y Perry, 2014).

El estudio de la transición entre etapas educativas, concretamente entre la Educación Infantil y la Educación Primaria, se erige como una temática de investigación importante debido a sus potenciales repercusiones, tanto positivas como negativas, en el marco de la primera transición educativa dentro de nuestro sistema educativo. En esta línea, el estudio de la transición entre las referidas etapas ha abordado el análisis de las diferencias o discontinuidades entre ellas, recogiendo la perspectiva de profesores y familias (Rimm-Kaufman y Pianta, 2000) e, incluso, la de los propios niños en cuanto que principales protagonistas de la transición (Babic, 2017; Castro, Ezquerra y Argos, 2015; Eskelä-Haapanen, Lerkkanen, Rasku-Puttonen y Poikkeus, 2016). También ha sido abordada la importancia del bienestar emocional durante el proceso de transición educativa (Bulkeley y Fabian, 2006; Kinkead-Clark, 2016), el estudio de la atención a la diversidad en el referido proceso (Dockett, Perry y Kearney, 2011; Janus, Lefort, Cameron y Kopechanski, 2007) o las expectativas que, tanto niños, como familias y profesorado poseen en torno al inicio de la 
A. CASTRO ZUBIZARRETA, P. EZQUERRA MUÑOZ Y J. ARGOS GONZÁLEZ

PROFUNDIZANDO EN LA TRANSICIÓN ENTRE EDUCACIÓN INFANTIL Y EDUCACIÓN PRIMARIA...

escolaridad obligatoria (Dockett y Perry, 2009). Asimismo, también ha sido foco de atención de investigaciones la adquisición del rol como alumno y el sentimiento de sentirse preparado para la Educación Primaria (Broström, 2002; Carr, 2001; Dockett y Perry, 2014), tanto socialmente, al lograr el pequeño la interacción positiva con otros iguales y encontrar otros profesores que se convierten en nuevos referentes adultos, como académicamente, al adquirir habilidades instrumentales.

A pesar de que, tal y como hemos mostrado, existen diferentes investigaciones que abordan la transición educativa, consideramos que el estudio de los mecanismos o actuaciones que facilitan una transición sin problemas y la adaptación exitosa de los niños a la Educación Primaria es inexistente en nuestro país y está escasamente abordada en el panorama internacional. En este último marco se inscribe el estudio longitudinal desarrollado por Schulting, Malone y Dodge (2005) en Estados Unidos que, contando con la participación de 17.212 niños y 992 escuelas, concluye que aquellos niños de Educación Infantil que participaron en actividades de transición educativa desarrolladas por sus maestros se adaptaron mejor a la escolaridad obligatoria que aquellos otros que no habían estado implicados en ellas. Este estudio ha favorecido el desarrollo posterior de programas y políticas educativas en otros países como Australia, donde se ha elaborado un potente marco de trabajo sobre la transición a la Educación Primaria (Dockett y Perry, 2014), plasmado en The Early Years Learning Framework y en The National Quality Standard for Early Childhood Education and Care and School Age Care (National Quality Standard). Otros países que están orientando políticas educativas con programas o normativa específica sobre la transición son Suecia o Colombia. Por su parte, en nuestro país, su presencia en el marco legislativo regulador se limita a la consideración de la necesaria y adecuada coordinación entre ambas etapas educativas.

Consideramos que una transición exitosa requiere de articulación y atención a los diversos actores y escenarios, hecho que pone de manifiesto Unicef (2009), cuando en su manual Escuelas amigas de la infancia sostiene que la preparación para la escolaridad obligatoria requiere de la interacción positiva de alumnado, escuelas y familias, entendido desde un planteamiento ecológico en el que todos los contextos y agentes están interconectados y pueden apoyarse a lo largo del proceso de transición, para facilitar la adaptación del niño a la escolaridad obligatoria, superando el mero trasiego de una etapa escolar a otra.

\section{PROFESORADO Y FAMILIA: DOS MIRADAS ANTE EL MISMO PROCESO DE TRANSICIÓN}

Docentes y familias son dos agentes clave para conseguir una transición armoniosa entre etapas. Estudios como el desarrollado por Chan (2010) ponen de manifiesto que la existencia de fuertes relaciones entre la Escuela Infantil, la Escuela Primaria y las familias podría facilitar una adecuada transición escolar. Por su parte, Pianta y Kraft-Sayre (2003) constataron en su estudio la demanda, tanto de familias como de maestros, de prácticas de transición que enfatizasen la articulación entre etapas para dotar de coherencia el paso de una etapa educativa a otra. En este 
A. CASTRO ZUBIZARRETA, P. EZQUERRA MUÑOZ Y J. ARGOS GONZÁLEZ

sentido, Sakellariou y Sivropoulou (2010) sostienen que las prácticas o actividades de transición educativa no solo benefician a los niños, sino también a las familias, que ven rebajado su nivel de estrés y preocupación en torno a la transición escolar de sus hijos, y al propio profesorado de ambas etapas educativas, que reconoce tener un conocimiento mayor del trabajo que se realiza en cada etapa.

A pesar de estos beneficios potenciales, la realidad es que las prácticas de transición educativa en los centros escolares no son una constante, prevaleciendo la existencia de intentos individuales frente a los comunitarios. En todo caso, diferentes estudios, tanto nacionales como internacionales, apuntan que las familias demandan información y comunicación con el profesorado de sus hijos sobre la transición a la Escuela Primaria (Castro, Argos y Ezquerra, 2012; Janus, 2011; Yi Hung Lau, 2014), y que la perspectiva de ambos agentes sobre el proceso de transición educativa no es tan coincidente como debiera, lo que pone de relieve la necesidad de contrastar pareceres, inquietudes y expectativas que favorezcan la creación de un marco común de entendimiento en torno a lo que supone el proceso de transición educativa de los pequeños a la escolaridad obligatoria.

Algunos planteamientos divergentes entre los docentes de ambas etapas son los encontrados por Rimm-Kaufman y Pianta (2000) que, a través de una encuesta nacional en Estados Unidos, constataron que mientras que para los maestros de Primaria la principal dificultad que observaban en los niños al inicio de esta etapa era la necesidad de seguir órdenes, el planteamiento del profesorado de Educación Infantil ponía el acento en la importancia de lograr cotas de independencia y autonomía en los pequeños. Por su parte, en el contexto inglés, Hendy y Whitebread (2000) identificaron la diferente percepción del profesorado de ambas etapas educativas en torno a la adquisición de la independencia por parte de los niños, siendo esta prioritaria para el profesorado de Educación Infantil y entendiendo los maestros de Educación Primaria que es una destreza compleja que no se alcanza al inicio de la escolaridad obligatoria.

Por su parte, aun encontrando planteamientos diferentes, Lara-Cinisomo et al. (2008) apuntan la existencia de creencias compartidas entre los docentes de ambas etapas que giran en torno a la importancia de asegurar el bienestar emocional de los niños, su motivación y gusto por la escuela, el desarrollo de una adecuada adquisición de destrezas motoras y la iniciación de los niños en habilidades instrumentales básicas.

Finalmente, adentrándonos en el contexto familiar, también hemos encontrado en la revisión de la literatura que las familias tienden a otorgar más importancia que los docentes de Educación Infantil y Primaria a la adquisición por parte de los niños de habilidades académicas (lectura, escritura y cálculo) ante su incorporación a la Educación Primaria (Chan, 2012; Dockett y Perry, 2014).

Esta diversidad de planteamientos y prioridades ante una misma realidad educativa hace que queramos estudiar la perspectiva de ambos agentes educativos, familia y profesorado, sobre el fenómeno de la transición desde la Etapa de 
A. CASTRO ZUBIZARRETA, P. EZQUERRA MUÑOZ Y J. ARGOS GONZÁLEZ

PROFUNDIZANDO EN LA TRANSICIÓN ENTRE EDUCACIÓN INFANTIL Y EDUCACIÓN PRIMARIA...

Educación Infantil a la de Educación Primaria. Para ello, los objetivos que guiaron el presente estudio fueron los siguientes:

- Identificar el grado de importancia/trascendencia que conceden profesorado y familias a la transición educativa.

- Definir cuáles son las habilidades o destrezas que perciben familias y profesorado como necesarias en el niño para que este se adapte con éxito a la Educación Primaria.

- Identificar cuáles son los cambios que, desde la perspectiva de familias y del profesorado de ambas etapas educativas, perciben los niños ante su incorporación a la escolaridad obligatoria.

- Conocer cuáles son los mayores desafíos, desde la opinión de profesorado y familias, a los que el niño se enfrenta ante el inicio de la Educación Primaria y cuáles son aquellos elementos facilitadores de una adecuada transición educativa.

- Conocer los mecanismos, actuaciones y/o prácticas que se ponen en funcionamiento por parte de los centros escolares para favorecer la transición escolar de los niños. A su vez, identificar el grado de importancia/necesidad que conceden, tanto familias como profesorado, a diversas propuestas de actuaciones o prácticas desarrolladas para favorecer la transición educativa, así como el grado de viabilidad que conceden a las mismas.

\section{MARCO EMPÍRICO}

\subsection{Participantes}

En el estudio participaron 460 personas, familias y profesorado del último curso de Educación Infantil y de primero de Educación Primaria. Concretamente, 153 personas corresponden al colectivo de familias cuyos hijos estaban escolarizados en tercero de Educación Infantil, 231 al de familias cuyos hijos estaban escolarizados en el primer curso de Educación Primaria y 76 al de docentes. Se ha optado por un muestreo causal o incidental que tuvo como criterio la participación en el estudio de familias y de profesorado del colegios públicos y concertados de la Comunidad Autónoma de Cantabria, ubicados en entornos urbanos y rurales.

\subsection{Consideraciones éticas}

El estudio fue aprobado por la Comisión de Ética y Humanidades de la Universidad de Cantabria para su realización en el curso académico 2016/2017. Durante todo el proceso de investigación se tuvieron en cuenta las siguientes premisas: el consentimiento informado de las familias y del profesorado del centro, la participación voluntaria de los sujetos, el derecho de los participantes a recibir información 
A. CASTRO ZUBIZARRETA, P. EZQUERRA MUÑOZ Y J. ARGOS GONZÁLEZ

PROFUNDIZANDO EN LA TRANSICIÓN ENTRE EDUCACIÓN INFANTIL Y EDUCACIÓN PRIMARIA...

sobre los resultados obtenidos en el estudio y el tratamiento riguroso de los datos que nos aportaran, así como la confidencialidad de estos.

\subsection{Procedimiento}

El desarrollo del estudio se llevó a cabo conforme a la siguiente secuenciación procedimental:

- Primer acercamiento: Contacto telefónico con los directores de los posibles centros educativos colaboradores en el proyecto. En él, se les informó sobre el propósito del estudio y el equipo investigador participante, remitiéndoles a aquellos que estuvieron interesados un escrito detallado, a través de correo electrónico, en el que quedaba plasmado el sentido y finalidad del estudio, la confidencialidad de los datos y el anonimato de los centros y personas participantes.

- Segundo acercamiento: Obtención del consentimiento informado. En aquellos centros educativos donde la dirección consideró interesante su participación en el estudio, la investigadora principal se dirigió personalmente al centro y mantuvo una reunión con la dirección en la que se informó pormenorizadamente del estudio y, más en concreto, de los instrumentos de recogida de información. En esa reunión se entregó la hoja de consentimiento para participar, así como una circular informativa para las familias.

- Recogida de información: Los profesores tutores de Educación Infantil y de primero de Educación Primaria de los centros educativos participantes recogieron los cuestionarios cumplimentados por las familias que se entregaban en sobre cerrado y que fueron, posteriormente, entregados a la investigadora principal. Del mismo modo, desde los centros se hizo también entrega en sobres cerrados de los cuestionarios cumplimentados por el profesorado de ambas etapas educativas.

\subsection{Método}

El enfoque metodológico fue el cuantitativo, utilizándose la técnica del cuestionario. Esta técnica destaca por su fácil y rápida distribución y recogida de opiniones e información de los sujetos investigados. También ofrece uniformidad de medición debido a sus opciones estandarizadas y registros de respuestas, así como la posibilidad de llegar a una muestra amplia de población.

Se elaboraron para este estudio tres cuestionarios conformados, en su gran parte, por preguntas presentadas en una escala tipo Likert con cuatro opciones de respuesta (desde "nada importante» a "muy importante»; desde "nada de acuerdo" a "muy de acuerdo»...) y por algunas preguntas de respuesta dicotómica y abierta (estas últimas no consideradas en el presente trabajo). Uno de los cuestionarios se destinó al profesorado de Educación Infantil y Educación Primaria y otros dos a 
familias. De estos últimos, uno de ellos iba dirigido a las familias de niños y niñas que asisten a las aulas de 5 años de Educación Infantil y otro a las familias con niños en $1 .^{\circ}$ de Educación Primaria.

Los cuestionarios contemplan diez dimensiones de análisis que permiten la triangulación de la perspectiva del profesorado y de las familias. Han sido elaborados específicamente para este estudio tomando como referencia tanto aportaciones de investigaciones previas, algunas ya reseñadas con anterioridad en este trabajo, como estudios exploratorios desarrollados por parte del equipo investigador.

Estas dimensiones son las siguientes: (I) Aspectos contextuales de centro, (II) Situación de partida del niño, (III) Cambios más notables percibidos entre las etapas educativas, (IV) Elementos facilitadores de la transición educativa, (v) Destrezas básicas requeridas para favorecer la transición educativa de los niños, (VI) Desafíos a los que se enfrenta el niño, (VII) Conductas observadas en los niños, (VIII) Información facilitada sobre la transición entre etapas educativas, (IX) Expectativas/preocupaciones y demandas y (x) Actuaciones o prácticas desarrolladas para favorecer una transición suave o armoniosa. En este trabajo, por limitaciones de espacio, nos centraremos en las dimensiones III, IV, V, VI, IX y X.

Con la dimensión III se pretende identificar las diferencias más importantes que cada uno de los colectivos encuestados percibe entre la Educación Infantil y la Educación Primaria y que pueden propiciar que la transición entre dichas etapas educativas pueda ser entendida como una ruptura o bien como una continuidad. Con la dimensión IV pretendemos conocer cuáles son los elementos que, tanto familias como profesorado, consideran que se convierten en elementos facilitadores de la transición educativa y, por lo tanto, propician que esta sea más suave y adecuada. La dimensión v permitirá conocer cuáles son las destrezas o habilidades que, tanto docentes como familias de ambas etapas, consideran que el niño tiene que tener adquiridas ante su paso a la Educación Primaria. La dimensión vi analiza los desafíos a los que los colectivos señalan que se enfrentan los niños ante el inicio de la escolaridad obligatoria. Finalmente, con las dimensiones IX y x pretendemos conocer cómo se aborda la transición educativa desde los centros escolares, identificando en el caso de la dimensión IX si se recogen y dan respuesta a las expectativas/preocupaciones y demandas sobre la transición educativa por parte de los colectivos encuestados y, en el caso de la última dimensión, conocer cuáles son las actuaciones desarrolladas para favorecer la transición educativa y el grado de necesidad y de viabilidad que perciben en aquellas que no conocen o que no han sido desarrolladas por el centro.

Para la validación del contenido de los cuestionarios se contó con la participación de seis expertos en Educación Infantil y Primaria tanto nacionales como extranjeros. Los criterios de elección contemplados se sustentaron en el dominio de la temática de estudio (en el caso de los tres docentes universitarios) y en una experiencia docente superior a diez años (en el caso de los tres maestros vinculados al último curso de Educación Infantil y/o al primero de Educación Primaria). 
A. CASTRO ZUBIZARRETA, P. EZQUERRA MUÑOZ Y J. ARGOS GONZÁLEZ

A los evaluadores expertos se les aportó una planilla de evaluación siguiendo el modelo propuesto por Escobar-Pérez y Cuervo-Martínez (2008), que recogía los siguientes indicadores: suficiencia, claridad, coherencia y relevancia. De acuerdo con la opinión de los jueces se realizaron cambios en la redacción de las destrezas que los niños tienen que tener adquiridas para pasar a la Educación Primaria, así como en el apartado de mecanismos o actuaciones que se ponen en funcionamiento por parte de los centros escolares y de las familias para favorecer la transición escolar de los niños a la Educación Primaria. Los cambios sugeridos proponían la incorporación de una definición de cada destreza y mecanismo favorecedor de la transición con el objeto de que estos pudieran ser comprensibles y no dieran lugar a diferentes interpretaciones.

Realizados estos ajustes se procedió a una segunda ronda de revisión donde los jueces valoraron de forma positiva la claridad del contenido. El índice Kappa obtenido en esta segunda ronda en el cuestionario a familias de niños de 5 años fue de 0,70 , en el cuestionario a familias de $1 .^{\circ}$ de Primaria fue de $0,72 \mathrm{y}$ en el cuestionario destinado al profesorado de 0,75 .

Para el estudio de la fiabilidad del instrumento se utilizó el análisis de la consistencia interna (Alfa de Cronbach). Los resultados obtenidos en él fueron de 0,867 para el cuestionario de las familias de Educación Infantil, de 0,879 para el de las familias de Educación Primaria y de 0,861 para el del profesorado.

Los resultados que a continuación se presentan se corresponden con los obtenidos en la Comunidad Autónoma de Cantabria, aunque este estudio forma parte de una investigación hispano-portuguesa que está en curso y que se enmarca en un proyecto coordinado por la Universidad de Cantabria con la Escola Superior de Educação do Porto, cuyas investigadoras colaboraron en el diseño y validación del cuestionario para, con posterioridad, poder adaptarlo al idioma y contexto portugués. El análisis de los datos se realizó utilizando el paquete estadístico spss 18.0.

\section{RESUltados}

\subsection{Importancia o trascendencia que conceden familias y profesorado a las transiciones educativas}

En el estudio hemos considerado relevante identificar el grado de importancia que conceden los participantes a las diferentes transiciones educativas que viven y experimentan los niños a lo largo de su escolarización. Al respecto, hemos de señalar que los colectivos representados en la investigación (familias de niños de cinco años, familias de niños de $1 .^{\circ}$ de Primaria y profesorado de ambas etapas educativas) otorgan diferente grado de importancia a las distintas transiciones educativas. Concretamente, el 49,3\% de las familias de niños de Educación Infantil consideran que la transición desde el contexto familiar a la Escuela Infantil es nada o poco importante, frente a un $50,7 \%$ que la considera bastante o muy importante. Por su parte, el $62,4 \%$ de las familias que tienen a sus hijos escolarizados en el 
primer curso de Educación Primaria valoran la incorporación de los niños a la Escuela Infantil como nada o poco importante, en contraposición al 37,6\% que lo considera bastante o muy importante. La diferencia es altamente significativa $(\mathrm{p}<.001)$ respecto del parecer del profesorado, del que solo un 7,7\% considera que la incorporación de los niños a la Escuela Infantil es poco importante frente al $89,5 \%$ que le otorga gran importancia.

En relación con la transición a la Educación Primaria, el 45,6\% de las familias de niños de Educación Infantil señalan que es nada o poco importante, frente a un $54,4 \%$ que la entiende como bastante o muy importante. En el caso de las familias de niños de primero de Educación Primaria nos encontramos con que el 64,4\% considera que la transición a la escolaridad obligatoria es nada o poco importante, frente a un 35,6\% que la percibe como bastante o muy importante. Finalmente, solo el 13,2\% del profesorado considera poco importante la transición a la Educación Primaria y un $86,8 \%$ la percibe como bastante o muy importante. La diferencia de pareceres, como se puede apreciar, vuelve a ser altamente significativa $(\mathrm{p}<.001)$ entre los diferentes colectivos.

La transición a la Educación Secundaria es percibida por el 39,1\% de las familias de niños de Educación Infantil como nada o poco importante frente a un $60,9 \%$ que la entiende como una transición importante. En el caso de las familias de niños de primero de Primaria, el 34, 2\% se refiere a la transición a la Educación Secundaria como nada o poco importante a diferencia del $65,8 \%$ que la entiende como bastante o muy importante. Finalmente, el 18,7\% del profesorado valora dicha transición como nada o poco importante en contraposición al 81,3\% que la percibe como importante, manteniéndose la existencia de diferencias de valores significativos $(\mathrm{p}<.05)$ entre la perspectiva de familias y profesorado.

TABLA 1

IMPORTANCIA O TRASCENDENCIA CONCEDIDA A LAS TRANSICIONES EDUCATIVAS

\begin{tabular}{|l|c|c|c|c|}
\hline & \multicolumn{3}{|c|}{ Colectivo } & \\
\cline { 2 - 5 } & Familias & Familias 1. \\
$\mathbf{5}$ años & Primaria & Profesorado & \\
\cline { 2 - 5 } & Media & Media & Media & Sig. \\
\hline $\begin{array}{l}\text { Grado de de importancia de la transición } \\
\text { del contexto familiar a Educación Infantil }\end{array}$ & 2.52 & 2.60 & 2.85 & $* * * *$ \\
\hline $\begin{array}{l}\text { Grado de importancia de la transición de } \\
\text { Educación Infantil a Educación Primaria }\end{array}$ & 2.22 & 2.15 & 2.79 & $* * * *$ \\
\hline $\begin{array}{l}\text { Grado de importancia de la transición de } \\
\text { Educación Primaria a Educación Secundaria }\end{array}$ & 3.63 & 3.32 & 3.27 & $*$ \\
\hline
\end{tabular}

NOTA. ${ }^{*}$ Valores significativos $\mathrm{p}<.05 ;{ }^{* *}$ Valores muy significativos $\mathrm{p}<.01$; *** Valores altamente significativos $\mathrm{p}<.001$. 
Llama la atención comprobar la diferencia de percepción existente en relación con la importancia que conceden familias y profesorado a las transiciones educativas, siendo este hecho especialmente acusado en las primeras transiciones. En concreto, en la transición entre Educación Infantil y Educación Primaria, que es en la que centramos nuestro estudio, el hecho de que casi la mitad de las familias de Educación Infantil la consideren como poco o nada importante puede comportar en ellas una motivación escasa hacia su implicación en ese proceso.

\subsection{Destrezas y/o competencias que deberian tener adquiridas los niños ante su incorporación a Educación Primaria}

Familias y profesorado coinciden en señalar que la autonomía personal (95,4\% familias de Infantil, 93,4\% familias de Primaria y el 98,7\% del profesorado) y las habilidades comunicativas (95,4\% familias de Infantil, 90,3\% familias de Primaria y el 94,8\% del profesorado) son destrezas que el niño debiera poseer para su adecuada transición educativa a Primaria. Las familias de niños que asisten a primero de Educación Primaria y el profesorado comparten su visión sobre la importancia de que el niño tenga adquirida una competencia motora básica ante su incorporación a esta etapa (86,9\% familias de Primaria, 92\% profesorado), si bien las familias de niños de cinco años (85,5\%) destacan el trabajo colaborativo como la tercera destreza básica a adquirir por sus hijos ante su incorporación a Educación Primaria. Los resultados del Anova unifactorial entre-grupos señalan que la diferencia entre las puntuaciones medias de familias y profesorado es estadísticamente significativa en las destrezas/competencias que se muestran sombreadas en la siguiente tabla. 
A. CASTRO ZUBIZARRETA, P. EZQUERRA MUÑOZ Y J. ARGOS GONZÁLEZ PROFUNDIZANDO EN LA TRANSICIÓN ENTRE EDUCACIÓN INFANTIL Y EDUCACIÓN PRIMARIA...

TABLA 2

DESTREZAS Y/O COMPETENCIAS QUE DEBERÍAN TENER ADQUIRIDAS LOS NIÑOS ANTE SU INCORPORACIÓN A LA EDUCACIÓN PRIMARIA

\begin{tabular}{|c|c|c|c|c|}
\hline & \multicolumn{3}{|c|}{ COLECTIVO } & \\
\hline & $\begin{array}{c}\text { Familias } \\
5 \text { años }\end{array}$ & $\begin{array}{c}{\text { Familias } 10^{\circ}}_{\text {Primaria }} \\
\end{array}$ & Profesorado & \\
\hline & Media & Media & Media & Sig. \\
\hline $\begin{array}{l}\text { La autonomía personal es una destreza que } \\
\text { debería tener adquirida el niño ante la EP }\end{array}$ & 3.76 & 3.68 & 3.89 & $*$ \\
\hline $\begin{array}{l}\text { Las habilidades comunicativas son una } \\
\text { destreza que debería tener adquirida el niño } \\
\text { ante la EP }\end{array}$ & 3.56 & 3.44 & 3.50 & \\
\hline $\begin{array}{l}\text { La atención y concentración es una destreza } \\
\text { que debería tener adquirida el niño ante la } \\
\text { EP }\end{array}$ & 3.33 & 3.17 & 3.28 & \\
\hline $\begin{array}{l}\text { La resolución de problemas/conflictos es } \\
\text { una destreza que debería tener adquirida el } \\
\text { niño ante la EP }\end{array}$ & 3.11 & 2.90 & 3.09 & $*$ \\
\hline $\begin{array}{l}\text { La lectura es una destreza que debería tener } \\
\text { adquirida el niño ante la EP }\end{array}$ & 2.87 & 2.84 & 2.49 & $* *$ \\
\hline $\begin{array}{l}\text { La escritura es una destreza que debería } \\
\text { tener adquirida el niño ante la EP }\end{array}$ & 2.80 & 2.81 & 2.47 & $*$ \\
\hline $\begin{array}{l}\text { El cálculo es una destreza que debería tener } \\
\text { adquirida el niño ante la EP }\end{array}$ & 2.66 & 2.74 & 2.51 & \\
\hline $\begin{array}{l}\text { El trabajo individual es una destreza que } \\
\text { debería tener adquirida el niño ante la EP }\end{array}$ & 3.02 & 2.96 & 2.92 & \\
\hline $\begin{array}{l}\text { El trabajo colaborativo es una destreza que } \\
\text { debería tener adquirida el niño ante la EP }\end{array}$ & 3.42 & 3.17 & 3.16 & \\
\hline $\begin{array}{l}\text { La destreza motora es una destreza que } \\
\text { debería tener adquirida el niño ante la } \mathrm{EP}\end{array}$ & 3.26 & 3.25 & 3.25 & \\
\hline
\end{tabular}

NOTA. * Valores significativos $\mathrm{p}<.05 ;{ }^{* *}$ Valores muy significativos $\mathrm{p}<.01$; ***⿰冫⿰亅⿱丿丶丶 Valores altamente significativos $\mathrm{p}<.001$. No se presentan los resultados no significativos.

El análisis posterior de las diferencias de medias mediante la prueba de Tukey señala que los colectivos encuestados difieren entre sí en el valor que otorgan a la necesidad de una mayor autonomía por parte de los niños, ya que existen diferencias significativas entre el valor que asigna el profesorado a esta destreza y la puntuación inferior que conceden las familias de Educación Primaria $(\mathrm{p}<.05)$, a pesar de que ambos colectivos coinciden en indicar que se trata de una destreza básica a adquirir para la incorporación a la Educación Primaria.

Por su parte, las familias de niños de cinco años conceden una puntuación significativamente mayor que las de niños de primer curso de Educación Primaria a la 
A. CASTRO ZUBIZARRETA, P. EZQUERRA MUÑOZ Y J. ARGOS GONZÁLEZ

PROFUNDIZANDO EN LA TRANSICIÓN ENTRE EDUCACIÓN INFANTIL Y EDUCACIÓN PRIMARIA...

resolución de problemas como una de las destrezas a adquirir $(\mathrm{p}<.05)$. Por último, el profesorado concede una puntuación significativamente menor que las familias a la necesidad de que los niños tengan adquiridas competencias en relación con la lectura $(\mathrm{p}<.01)$ y la escritura $(\mathrm{p}<.05)$ ante el inicio de la escolaridad obligatoria. Estas discrepancias, especialmente las relacionadas con la competencia lectora, pueden comportar la existencia de una cierta incomprensión familiar acerca del trabajo a desarrollar por los docentes en cada una de las etapas. Dicho desacuerdo, afortunadamente, no se constata en relación con otras competencias de carácter no instrumental, como la autonomía personal o las habilidades comunicativas.

\subsection{Cambios percibidos y desafíos a los que se enfrentan los niños ante el inicio de la Educación Primaria}

Analizando las percepciones de familias y profesorado, los resultados de los cuestionarios ponen de manifiesto que el $69.23 \%$ de las familias considera que existen diferencias notables entre ambas etapas educativas. Comprobamos que un $72.60 \%$ de las familias de niños de cinco años percibe diferencias importantes al igual que el $67.10 \%$ de las familias de niños que ya se encuentran en la Educación Primaria.

Al preguntar al profesorado si concibe la transición entre la Educación Infantil y la Educación Primaria como una ruptura o como una continuidad educativa constatamos que el 31.6\% entiende que la transición a la Educación Primaria supone una ruptura educativa mientras que el $68.4 \%$ la percibe como una continuidad.

En relación con la percepción sobre los desafíos a los que se enfrentan los niños, tanto familias como profesorado coinciden en señalar que los principales desafíos ante la transición a la Educación Primaria se encuentran en la adquisición de aprendizajes relacionados con las habilidades instrumentales básicas $(90.6 \%$ familias de Infantil, $89 \%$ familias de Primaria y $76.3 \%$ profesorado) y con el cambio en la forma de trabajar en clase (87.2\% familias de Infantil, $78 \%$ familias de Primaria y $76.3 \%$ profesorado).

Los resultados del Anova unifactorial entre-grupos señala que existen diferencias estadísticamente significativas entre las puntuaciones medias de familias y profesorado en diferentes variables. 
TABLA 3

DESAFÍOS A LOS QUE SE ENFRENTAN LOS NIÑOS ANTE EL INICIO DE LA EDUCACIÓN PRIMARIA

\begin{tabular}{|l|c|c|c|c|}
\hline \multirow{2}{*}{} & \multicolumn{3}{|c|}{ COLECTIVO } & \\
\cline { 2 - 5 } & $\begin{array}{c}\text { Familias } \\
\text { 5 años }\end{array}$ & $\begin{array}{c}\text { Familias 1. } \\
\text { Primaria }\end{array}$ & Profesorado & \\
\cline { 2 - 5 } & Media & Media & Media & Sig. \\
\hline Una mayor autonomía & 3.15 & 3.21 & 2.76 & $* * * *$ \\
\hline El cambio en la forma de trabajar en la clase & 3.33 & 3.11 & 3.05 & $* * *$ \\
\hline $\begin{array}{l}\text { La necesidad de resolver por sí mismo } \\
\text { ciertos conflictos cotidianos }\end{array}$ & 3.05 & 3.02 & 2.55 & $* * * *$ \\
\hline $\begin{array}{l}\text { La adquisición de aprendizajes relacionados } \\
\text { con la lectura, la escritura y el cálculo }\end{array}$ & 3.39 & 3.41 & 3.24 & \\
\hline La adaptación a nuevos profesores & 3.28 & 2.85 & 2.61 & $* * * *$ \\
\hline La adaptación a nuevos espacios & 2.95 & 2.56 & 2.64 & $* * * *$ \\
\hline $\begin{array}{l}\text { La necesidad de hacer nuevos amigos, } \\
\text { debido al cambio de compañeros en el } \\
\text { grupo }\end{array}$ & 2.60 & 2.41 & 2.05 & $* * * * *$ \\
\hline Apuntar y recordar las tareas & 3.12 & 3.11 & 2.89 & \\
\hline La adaptación a nuevas rutinas & 3.12 & 2.95 & 2.84 & $*$ \\
\hline $\begin{array}{l}\text { Permanecer tiempos prolongados sentados } \\
\text { y prestar atención }\end{array}$ & 3.26 & 3.11 & 3.44 & $* * *$ \\
\hline
\end{tabular}

NOTA. * Valores significativos $\mathrm{p}<.05 ; *$ Valores muy significativos $\mathrm{p}<.01$; *** Valores altamente significativos $\mathrm{p}<.001$. No se presentan los resultados no significativos.

El análisis posterior de las diferencias de medias mediante la prueba de Tukey indica que los colectivos encuestados difieren entre sí al considerar los desafíos a acometer. En el relativo a la necesidad de una mayor autonomía por parte de los niños, es el profesorado quien lo percibe como un elemento de menor importancia en contraste con la percepción de las familias de ambas etapas educativas ( $\mathrm{p}<$ .001). Esta situación se repite en lo referido a la necesidad de resolver conflictos por sí mismos $(\mathrm{p}<.001)$ y a la adaptación a nuevos profesores $(\mathrm{p}<.001)$. Del mismo modo, también difiere su percepción en relación con el cambio en la forma de trabajar en clase $(\mathrm{p}<.05)$, aun siendo este, como ya hemos señalado, uno de los principales desafíos contemplados por los diferentes colectivos.

La adaptación a los nuevos espacios es un desafío que las familias de niños de cinco años perciben de forma más intensa que el profesorado y que las familias de Educación Primaria $(\mathrm{p}<.001)$. La necesidad de hacer nuevos amigos también es concebida como un desafío para los niños, de mayor calado para las familias con hijos en las aulas de Educación Infantil que para el profesorado $(\mathrm{p}<.001)$ y, 
A. CASTRO ZUBIZARRETA, P. EZQUERRA MUÑOZ Y J. ARGOS GONZÁLEZ

PROFUNDIZANDO EN LA TRANSICIÓN ENTRE EDUCACIÓN INFANTIL Y EDUCACIÓN PRIMARIA...

en menor medida, para las familias de Educación Primaria que para los docentes $(\mathrm{p}<0.05)$. También encontramos diferencias significativas $(\mathrm{p}<0.05)$ entre las familias de Educación Infantil y el profesorado en relación con la adaptación de los pequeños a nuevas rutinas.

Finalmente, nos parece interesante señalar que es el profesorado quien percibe como un desafío importante el que los niños deban de permanecer tiempos prolongados sentados y prestar atención, en contraste con la puntuación otorgada por las familias de ambas etapas educativas $(\mathrm{p}<.01)$.

De los datos anteriores nos parece significativo destacar el hecho de que tanto familias como profesorado coincidan en contemplar como importantes dos desafíos: el referido a la adquisición de aprendizajes instrumentales y el que atiende a los cambios en la forma de trabajar en clase. Esta coincidencia de pareceres, indudablemente, puede favorecer el desarrollo de una práctica pedagógica consistente y armónica, favorecedora de una mejor adaptación de los niños a la nueva etapa educativa.

Otra cuestión de interés está relacionada con la consideración de la autonomía de los pequeños como un desafío a contemplar. En este sentido, se pone de manifiesto una situación que podría parecer, a priori, un tanto paradójica. Así, mientras que en el epígrafe anterior constatábamos que los docentes la valoraban como una competencia muy relevante que deberían de tener adquirida los niños ante su incorporación a la Educación Primaria, no la contemplan con tanta relevancia en cuanto que desafío al que se enfrentan los pequeños. Esta valoración menor puede deberse al hecho de que el profesorado entiende que pueden tenerla ya moderadamente adquirida al iniciar la nueva etapa educativa.

\subsection{Elementos facilitadores de la transición educativa a la Educación Primaria}

Entendemos los facilitadores como aquellos elementos, circunstancias o actuaciones que favorecen una adecuada transición. Los tres elementos que son percibidos como los mayores facilitadores de la transición educativa para los colectivos encuestados son los siguientes.

Las familias de niños de cinco años identifican como el principal facilitador la coordinación entre la Educación Infantil y Primaria (98.7\%); en segundo lugar, el adecuado seguimiento del niño en el contexto escolar (92.8\%), y, en tercero, la existencia de un programa de transición educativa elaborado desde el centro (92.8\%).

Por su parte, las familias de Educación Primaria señalan como mayor facilitador la coordinación entre Educación Infantil y Primaria (94.7\%), el adecuado seguimiento del niño en el contexto escolar (93.4\%) y el mantenimiento de las relaciones de amistad (88.2\%).

Finalmente, el profesorado identifica, al igual que las familias, como el mayor facilitador la buena coordinación entre la Educación Infantil y la Educación Primaria (98.7\%) y el adecuado seguimiento del niño en el contexto escolar (88\%) pero, a diferencia de las familias, señala en tercer lugar la asistencia previa a la Educación Infantil (82.8\%). 
Este análisis pone de relieve el consenso existente entre los colectivos encuestados sobre el valor que conceden a una buena coordinación entre etapas educativas y al seguimiento del niño en el contexto escolar, si bien, se apuntan percepciones idiosincráticas de cada colectivo, dependiendo de los diferentes elementos contemplados.

Los resultados del Anova unifactorial entre-grupos señalan que la diferencia entre las puntuaciones medias de familias y profesorado es estadísticamente significativa en los elementos facilitadores sombreados en la tabla siguiente.

TABLA 4

ELEMENTOS FACILITADORES DE LA TRANSICIÓN A LA EDUCACIÓN PRIMARIA

\begin{tabular}{|c|c|c|c|c|}
\hline & \multicolumn{3}{|c|}{ COLECtivo } & \\
\hline & $\begin{array}{l}\text { Familias } \\
5 \text { años }\end{array}$ & $\begin{array}{c}{\text { Familias } 10^{\circ}}^{\text {Primaria }} \\
\end{array}$ & Profesorado & \\
\hline & Media & Media & Media & Sig. \\
\hline $\begin{array}{l}\text { Tener hermanos mayores que están en Ed. } \\
\text { Primaria }\end{array}$ & 2.84 & 2.64 & 2.77 & \\
\hline El mes de nacimiento del niño & 2.71 & 2.61 & 2.26 & *** \\
\hline $\begin{array}{l}\text { Un adecuado seguimiento del niño en el } \\
\text { contexto escolar }\end{array}$ & 3.50 & 3.48 & 3.53 & \\
\hline Asistencia previa a la Educación Infantil & 3.41 & 3.29 & 3.37 & \\
\hline $\begin{array}{l}\text { Conocimiento previo del espacio escolar } \\
\text { (visitas puntuales) }\end{array}$ & 3.18 & 3.11 & 3.03 & \\
\hline $\begin{array}{l}\text { Compartir ambas etapas educativas el mismo } \\
\text { recinto (instalaciones comunes) }\end{array}$ & 3.19 & 3.18 & 3.09 & \\
\hline $\begin{array}{l}\text { Compartir ambas etapas educativas el } \\
\text { mismo edificio }\end{array}$ & 2.87 & 2.92 & 2.84 & \\
\hline $\begin{array}{l}\text { Conocimiento previo del profesorado de la } \\
\text { nueva etapa educativa }\end{array}$ & 3.20 & 2.93 & 2.99 & $*$ \\
\hline $\begin{array}{l}\text { Mantenimiento de las relaciones de amistad } \\
\text { del alumnado }\end{array}$ & 3.39 & 3.34 & 3.15 & $*$ \\
\hline $\begin{array}{l}\text { Mantenimiento del grupo-clase de Educación } \\
\text { Infantil }\end{array}$ & 3.22 & 3.11 & 2.64 & $* * *$ \\
\hline $\begin{array}{l}\text { Información al niño sobre las rutinas } \\
\text { escolares en Educación Primaria }\end{array}$ & 3.28 & 3.21 & 3.16 & \\
\hline $\begin{array}{l}\text { Un programa de transición educativa } \\
\text { elaborado desde el centro }\end{array}$ & 3.44 & 3.20 & 3.46 & $* *$ \\
\hline $\begin{array}{l}\text { Una buena coordinación entre Educación } \\
\text { Infantil y Primaria }\end{array}$ & 3.69 & 3.62 & 3.83 & $*$ \\
\hline
\end{tabular}

NOTA. * Valores significativos $\mathrm{p}<.05$; *** Valores muy significativos $\mathrm{p}<.01$; **** Valores altamente significativos $\mathrm{p}<.001$. No se presentan los resultados no significativos. 
El análisis posterior de las diferencias de medias mediante la prueba de Tukey indica que los colectivos encuestados difieren entre sí de forma significativa en el grado de ayuda que suponen para la transición educativa una serie de elementos.

En primer lugar, las familias de ambas etapas perciben, en mayor medida que el profesorado, el mes de nacimiento como un elemento facilitador de la transición $(\mathrm{p}<.01)$.

Por su parte, las familias de niños de cinco años entienden que el conocimiento que tengan los niños del profesorado de la nueva etapa educativa es un facilitador importante, siendo la puntuación que otorgan a esta variable significativamente mayor $(\mathrm{p}<.01)$ que la de las familias de Primaria y el profesorado. A su vez, las familias conceden mayor importancia que el profesorado $(\mathrm{p}<.05)$ al mantenimiento de las relaciones de amistad.

Asimismo, se encuentran diferencias muy significativas de percepción entre los colectivos en relación con el mantenimiento del grupo-clase de Educación Infantil, sobre todo entre el profesorado y las familias ( $<<.001)$, mostrando los docentes su predisposición a la configuración de nuevos grupos-clase en Educación Primaria.

También se constatan diferencias significativas entre el valor que conceden las familias de Educación Infantil y profesorado a la existencia de un programa de transición educativa como facilitador de la misma, respecto de las familias de Educación Primaria $(\mathrm{p}<.01)$.

Finalmente, la buena coordinación entre la etapa de Educación Infantil y Primaria es percibida como un elemento facilitador al que conceden gran importancia todos los colectivos encuestados, encontrándose diferencias significativas $(\mathrm{p}<.05)$, entre las familias de Educación Primaria y el profesorado.

La elevada valoración media obtenida por la mayoría de los elementos facilitadores sometidos a valoración pone de manifiesto la relevancia de todos ellos de cara a la generación de un adecuado proceso de transición y, consiguientemente, la necesidad de tenerlos en cuenta por parte de los diferentes agentes educativos implicados.

\subsection{Expectativas, preocupaciones y demandas}

Nos parece reseñable el hecho de que un 57.2\% de los participantes tengan claro que sería necesario desarrollar un programa de transición educativa en su centro, mientras que un $21.5 \%$ señalen que no es necesario. Estos datos positivos en torno a la necesidad del desarrollo de un programa de transición educativa en los centros son coherentes con los datos presentados en el epígrafe anterior, donde todos los colectivos encuestados identificaban que la existencia de dicho programa, elaborado desde el centro, era un elemento facilitador de la transición para los niños (92.8\% familias Infantil; 82.9\% familias de Primaria y 88\% profesorado).

En otro orden de cosas, llama la atención que, mientras que el 80\% de docentes señala que las familias les demandan información sobre la transición educativa 
A. CASTRO ZUBIZARRETA, P. EZQUERRA MUÑOZ Y J. ARGOS GONZÁLEZ

PROFUNDIZANDO EN LA TRANSICIÓN ENTRE EDUCACIÓN INFANTIL Y EDUCACIÓN PRIMARIA...

a Primaria, solo el $47.8 \%$ de estas indica que haya hecho llegar al profesorado sus preocupaciones o expectativas sobre dicha transición.

A su vez, también hemos querido indagar si existen demandas de información por parte de los niños a sus referentes adultos (familia y profesorado) acerca de la transición educativa. En este sentido, el $65.25 \%$ de las familias considera que los niños no demandan información sobre la transición educativa frente a un $27.59 \%$ que sí lo hacen y un $7.16 \%$ que desconoce si su hijo ha solicitado información. Por su parte, el 56\% del profesorado señala que los niños demandan información sobre la transición, frente a un 36\% que expresa no haber tenido dicha demanda y un $8 \%$ que desconoce si los niños lo han hecho.

Resulta un tanto llamativo que sea el colectivo de docentes quien constate una mayor demanda de información por parte de los niños y que solo un $27.59 \%$ de las familias manifiesten recibir comentarios de sus hijos preguntando sobre la transición a la Educación Primaria. Tal vez, el hecho de que demanden más información a los docentes que a las familias pueda deberse a que son cuestiones que los pequeños vinculan al ámbito escolar, más allá de la estrecha relación que los niños de estas edades mantienen con sus familias o, tal vez, a que, dado que las familias conceden escasa importancia al proceso de transición, tienden a prestar poca atención a sus hijos cuando les demandan información.

Esta constatación es coherente con lo apuntado anteriormente en relación con la escasa importancia concedida por las familias al proceso de transición entre Educación Infantil y Educación Primaria. Así, si los niños demandan escasa información a sus familias acerca de dicho proceso, estas pueden no ser muy conscientes de su relevancia.

\subsection{Actuaciones y prácticas que se desarrollan por parte de los centros escolares y de las familias para favorecer la transición escolar de los niños a la Educación Primaria}

Nuestro marco legislativo apela a la necesaria coordinación entre etapas educativas para favorecer los procesos de transición, por lo que hemos preguntado al profesorado cuál es su percepción sobre la coordinación existente entre la Educación Infantil y Primaria. Un 36.8\% de docentes responde a esta cuestión señalando que es escasa o inexistente, siendo el profesorado que tiene más de seis años de experiencia el que valora mejor la coordinación respecto del que cuenta con menos de cinco años $(\mathrm{p}<.01)$. Sin embargo, no encontramos diferencias en cuanto a variables de centro, como su titularidad o a la etapa educativa en la que imparte docencia el profesorado.

Nos ha parecido relevante conocer las actuaciones y prácticas que se ponen en funcionamiento por parte de los centros escolares para favorecer la transición escolar de los niños a la Educación Primaria, cotejando la perspectiva de los tres colectivos. A su vez, hemos pretendido conocer la importancia que conceden a 
cada una de las prácticas que se implementan y a la viabilidad que otorgan a las mismas.

En relación con la puesta en práctica de diferentes actuaciones, el análisis de los datos indica que las reuniones al inicio de curso (91.2\% familias Infantil, $91.4 \%$ familias Primaria y $90,5 \%$ profesorado), los intentos del profesorado por responder a las inquietudes y preguntas de las familias (91.4\% familias Infantil, 91\% familias Primaria y $83.8 \%$ profesorado) y las visitas del profesorado de Educación Infantil con sus alumnos al edificio de Educación Primaria (90.5\% profesorado) son las actividades más consolidadas en los centros escolares.

Por su parte, las actividades menos habituales en los centros son los consejos de los niños de Primaria a los niños que van a iniciar esta etapa educativa $(28.5 \%$ profesorado), la presentación del profesorado de Educación Primaria a los niños de Educación Infantil y a sus familias (26.9\% familias Infantil, 39\% familias Primaria y 21.3\% profesorado), la entrega de información por escrito a las familias sobre la transición educativa (30.8\% familias Infantil, 51.7\% familias Primaria y 34.2\% profesorado) y la realización de actividades/proyectos conjuntos entre Educación Infantil y Primaria (51.4\% familias Infantil, 50.2\% familias Primaria y 62.7\% profesorado).

Resulta estadísticamente significativa la diferencia de percepción que tienen familias y profesorado en relación con la recogida, por parte de los docentes, de las inquietudes y preguntas de las familias sobre la Educación Primaria. Las familias perciben en mayor medida que el profesorado que este recoge sus preguntas e inquietudes $(\mathrm{p}<.05)$, hecho que resulta un tanto sorprendente. Tal vez, los docentes no sean conscientes de que, en la dinámica cotidiana y de manera informal, pueden estar aportando dicha información.

Asimismo, encontramos diferencias de percepción entre ambos colectivos acerca de la actividad en la que el profesorado de Educación Primaria se presenta a las familias y niños de Educación Infantil. Aunque tanto el profesorado como las familias señalan que esta no es una práctica extendida en las escuelas, la consideración sobre su necesidad y viabilidad difiere entre ambos colectivos. Así, mientras que las familias entienden que es una actividad necesaria y viable, los docentes manifiestan serias dudas sobre su viabilidad. Esta discrepancia puede responder al desconocimiento que tienen las familias respecto del profesorado interino (no fijo), que provoca inestabilidad de la plantilla y que influye en que el profesorado estime que esta actividad es poco viable.

En lo que respecta a la necesidad de las diferentes actuaciones, las familias de ambas etapas educativas coinciden en señalar las siguientes como las más necesarias: que el profesorado intente responder a las preguntas de las familias $(90.8 \%$ familias Infantil, 88.5\% familias Primaria), las reuniones al inicio de curso (99\% familias Infantil, $96.2 \%$ familias Primaria) y la reunión con familias para aportar información en el último trimestre de Educación Infantil (93.2\% familias Infantil; 90.2\% familias Primaria).

Por su parte, el profesorado recoge como actividades más necesarias que los docentes de ambas etapas educativas se reúnan para comentar informes de 
A. CASTRO ZUBIZARRETA, P. EZQUERRA MUÑOZ Y J. ARGOS GONZÁLEZ

PROFUNDIZANDO EN LA TRANSICIÓN ENTRE EDUCACIÓN INFANTIL Y EDUCACIÓN PRIMARIA...

seguimiento de cada niño antes del inicio del curso escolar (98.5\%), la reunión con las familias al inicio de curso $(96.2 \%)$ y la reunión con familias para aportar información en el último trimestre de la etapa de Educación Infantil (88.9\%).

\section{CONCLUSIONES}

La transición a la Educación Primaria es un acontecimiento único e irrepetible, diacrónico en el tiempo, que precisa de una atención especial dada la repercusión que la vivencia de una transición exitosa por parte de los niños tiene en el futuro rendimiento escolar y en la adaptación social de los pequeños (Featherstone, 2004).

Una adecuada transición escolar requiere de un planteamiento ecológico del proceso, en el que todos los contextos y agentes estén interconectados, sean interdependientes y puedan apoyarse, de manera que faciliten la adaptación del niño a la escolaridad obligatoria.

Dicha colaboración y apoyo precisa, en primer lugar, conocer las creencias y las perspectivas que tanto familias como profesorado poseen sobre el proceso de transición para, a partir de ellas, generar un marco común de entendimiento que favorezca la construcción de auténticas transiciones educativas a la escolaridad obligatoria, que sean armoniosas y que se lleven a cabo sin sobresaltos ni desafíos inalcanzables para los pequeños. En este sentido, coincidimos con el planteamiento de Unicef (2009) y con Fabian y Dunlop (2012) al entender que, para que niños, familias y escuelas estén preparados para la transición, se ha de comenzar por identificar cuál es el estado de la transición educativa en las escuelas.

En nuestro estudio se ha puesto de manifiesto la existencia de una brecha importante de percepción entre profesorado y familias en torno a la trascendencia concedida a las transiciones que se viven en la primera infancia. Hemos constatado que las familias no perciben como importantes las primeras transiciones educativas, lo que nos conduce a la necesidad de sensibilizarlas sobre la importancia y las repercusiones que puede tener para sus hijos una adecuada transición. Al respecto, Doucet y Tudget (2007) y Kaplun, Dockett y Perry (2017) apuntan en sus estudios que la concepción que las familias tienen de la transición educativa y los mensajes que transmiten a sus hijos dependen, en gran medida, de sus experiencias previas. En este sentido, las familias centran sus recuerdos de sus transiciones educativas en la posible experimentación de situaciones de bajo rendimiento o fracaso escolar al final de la etapa de Educación Primaria y en la etapa de Educación Secundaria y no a su inicio, obviando que es la primera transición educativa la que determina el futuro rendimiento escolar y la adaptación social de los pequeños (Featherstone, 2004). Este argumento puede ayudarnos a comprender por qué las familias otorgan en nuestro estudio mayor trascendencia a las transiciones hacia la Educación Secundaria.

A pesar de esta discrepancia, se aprecian puntos de encuentro entre las perspectivas del profesorado y de las familias de ambas etapas educativas. Tanto estas como los docentes destacan que la autonomía personal y las habilidades 
comunicativas son destrezas que los niños debieran tener adquiridas al inicio de la escolaridad obligatoria. Este consenso en relación a estas destrezas coincide con los resultados ya apuntados en el estudio de Castro, Ezquerra y Argos (2012).

Por su parte, encontramos desencuentros o pensamientos divergentes entre familias y docentes en torno al grado de importancia que conceden a la adquisición, por parte de los niños, de habilidades académicas (lectura, escritura y cálculo). En este sentido, las familias otorgan más importancia que el profesorado a su adquisición, algo que coincide con lo identificado por autores como Chan (2012), Dockett y Perry (2014) y Kinkead-Clark (2015), llegando a señalar Yi Hung Lau (2014) que las familias trabajan en casa estas actividades académicas para preparar bien a sus hijos. Este hecho pone de relieve que no existe un acuerdo claro entre lo que familias y profesorado entienden por "estar preparado» para la Educación Primaria, tanto en nuestro contexto como en el ámbito internacional. En todo caso, en lo que sí coinciden familias y profesorado es en percibir como principales desafíos de la transición a la Educación Primaria la adquisición de aprendizajes relacionados con las habilidades instrumentales básicas y con el cambio en la forma de trabajar en clase. De ello podemos inferir que las familias consideran conveniente anticipar la adquisición de habilidades de lectoescritura en los últimos cursos de Educación Infantil, mientras que el profesorado entiende que esta etapa ha de contemplar solo una aproximación a estas habilidades.

Asimismo, el estudio pone de manifiesto que las familias perciben cambios o discontinuidades importantes entre la Educación Infantil y la Educación Primaria. También resulta significativo que casi un tercio del profesorado entienda que la transición a la Educación Primaria supone una ruptura educativa, lo que evidencia la necesidad de explicar estos cambios tanto a las familias como a los niños, principales protagonistas de la transición educativa. En este sentido, Zhao (2017) insiste en la importancia de anticipar a los pequeños y a sus familias el conocimiento de las reglas y normas que rigen el funcionamiento de la Educación Primaria.

Por otro lado, es destacable que sea el colectivo de familias de niños de Educación Infantil el que conceda más importancia al conocimiento de los nuevos espacios en los que se van a desenvolver sus hijos, así como al mantenimiento de los vínculos de amistad que han creado durante esta etapa educativa.

Son las familias de los niños que van a incorporase a la Educación Primaria las que parecen estar más próximas a los planteamientos señalados por Bulkeley y Fabian (2006) y por Kinkead-Clark (2016), quienes inciden en la importancia de velar por el bienestar emocional de los alumnos facilitando, en primer lugar, el conocimiento de los nuevos espacios y, en segundo término, la creación de sentimientos de pertenencia y de identificación con la etapa Primaria. Asimismo, estos autores consideran que sería recomendable mantener los vínculos de amistad, ya que son entendidos como apoyos para afrontar el cambio. Es por ello por lo que creemos que, si bien el profesorado encuestado no considera importante el mantenimiento del grupo-clase de Educación Infantil para el tránsito a la Educación 
Primaria, debería tener en cuenta en la configuración de los nuevos agrupamientos el mantenimiento, en la medida de lo posible, de los vínculos de amistad ya existentes entre el alumnado.

Como elementos facilitadores de la transición educativa, recurrentes en la percepción de familias y profesorado, se han identificado la coordinación entre la Educación Infantil y Primaria y el adecuado seguimiento de los niños en el contexto escolar. Resulta llamativo que más de un tercio del profesorado considere que la coordinación entre etapas educativas es escasa o inexistente en su centro y que algo más de la mitad de los participantes en el estudio reclamen la existencia de un programa de transición educativa.

Otros datos que nos pueden ayudar a reflexionar sobre la importancia de esta temática son que mientras que una gran mayoría del profesorado señala que las familias le demandan información sobre la transición educativa a Educación Primaria, no llega a la mitad el número de familias que reconozca que traslada al profesorado sus preocupaciones y expectativas reales sobre la transición de sus hijos. Este dato remarca la importancia de tejer una adecuada relación familiaescuela que se sustente en una comunicación fluida y de confianza. Una situación coincidente con lo apuntado por varios estudios internacionales que indican que las familias demandan más comunicación e información por parte de los docentes para ayudar a sus hijos, así como oportunidades para participar y colaborar con el centro educativo (Janus, 2011; Yi Hung Lau, 2014).

Por otro lado, de los resultados que arroja el estudio acerca de la comunicación de los adultos con los niños sobre sus expectativas y demandas de información sobre la transición educativa a Primaria se infiere que las familias, al concebir la transición a la Educación Primaria como un proceso de escasa importancia, tienden a no prestar atención a las posibles demandas de información que plantean sus hijos (Kaplun, Dockett y Perry, 2017).

La constatación de los hallazgos aquí plasmados nos ha de animar a la generación de programas de transición educativa adaptados a las necesidades y demandas de cada centro escolar, que favorezcan la participación de todos los agentes implicados, entre ellos los propios niños, con su protagonismo en prácticas como los consejos que pueden aportar los niños de Primaria a los de último curso de Educación Infantil, que hemos comprobado que son casi inexistentes en los centros escolares.

En todo caso, también apuntamos la necesidad de generar una propuesta global y ecológica para el tratamiento de la transición educativa que contemple desde aspectos ligados al macrocontexto (referentes a políticas educativas orientadoras de transiciones adecuadas), al mesocontexto (relacionados con la comunicación y colaboración entre el profesorado de ambas etapas educativas y las familias) y, por último, prácticas y actuaciones en las aulas escolares, que serían el microcontexto en el que se evidencian e implementan dichas prácticas para favorecer la transición educativa deseable. 
A. CASTRO ZUBIZARRETA, P. EZQUERRA MUÑOZ Y J. ARGOS GONZÁLEZ

PROFUNDIZANDO EN LA TRANSICIÓN ENTRE EDUCACIÓN INFANTIL Y EDUCACIÓN PRIMARIA...

\section{REFERENCIAS BIBLIOGRÁFICAS}

ABello, R. (2008) Informe de Tesis Doctoral. Transiciones al Inicio de la Escolaridad: Una experiencia de construcción de sentido. Doctorado en Ciencias Sociales Niñez y Juventud CINDE-Universidad de Manizales.

BABIĆ, N. (2017) Continuity and discontinuity in education: example of transition from preschool to school. Early Child Development and Care, 187 (10), 1596-1609.

Brostrom, S. (2002) Communication and continuity in the transition from kindergarten to school, en Fabian, H. y Dunlop, A-W. (eds.) Transitions in the early years: Debating continuity and progression for children in early education. London, Routledge Falmer, $52-63$

Bulkeley, J. y FABIAN, H. (2006) Well-being and belonging during early educational transitions. International Journal of Transitions in Childhood, 2, 18-31.

CARr, M. (2001) Assessment in early childhood settings, Learning Stories. London, Paul Chapman.

CAstro, A.; Argos, J. y EzQuerra, P. (2012) La transición entre la escuela de educación infantil y la de la educación primaria: perspectivas de niños, familias y profesorado. Revista Española de Pedagogía, 70 (253), 537-552.

Castro, A.; EzQuerra, P. y Argos, J. (2015) La mirada infantil sobre el proceso de transición escolar desde la etapa de educación infantil a la de educación primaria. Perfiles Educativos, XXXVII, 24-49.

CHAN, W. L. (2010) The transition from kindergarten to primary school, as experienced by teachers, parents and children in Hong Kong. Early Child Development and Care, 180 (7), 973-993.

CONYERS, L. M.; ReYNolds, A. J. y Ou, S. R. (2003) The effect of early childhood intervention and subsequent special education services: Findings from the Chicago child-parent centers. Educational Evaluation and Policy Analysis, 25 (1), 75-95.

Dockett, S. y Perry, B. (2009) Readiness for school: a relational construct. Australasian Journal of Early Childhood, 34 (1), 20-26.

DOCKeTT, S. y PERRY, B. (2014) Continuity of Learning: A resource to support effective transition to school and school age care. Canberra, ACT: Australian Government Department of Education.

Dockett, S.; Perry, B. y Kearney, E. (2011) Starting School with Special Needs: Issues for Families with Complex Support Needs as Their Children Start School. Exceptionality Education International, 21, 45-61.

Doucet, F. y Tudge, J. (2007) Co-Constructing the transition to school: Reframing the "novice» versus «expert» roles of children, parents, and teachers from a cultural perspective, en PIANTA, R. C.; CoX, M. J. y SNOw, K. (eds.) School readiness and the transition to kindergarten in the era of accountability. Baltimore, MD, Brookes Publishing, 307-328.

Escobar-Pérez, J. y Cuervo-MarTínez, A. (2008) Validez de contenido y juicio de expertos: una aproximación a su utilización. Avances en Medición, 6 (1), 27-36.

eskelä-HaApanen, S.; Lerkkanen, M-K.; Rasku-Puttonen, H. y Poikkeus, A-M. (2016) Children's beliefs concerning school transition. Early Child Development and Care, 187 (9), 1446-1459.

Fabian, H. y DunloP, A. W. (eds.) (2002) Transitions in the Early Years. Debating continuity and progression for children in early education. London, Routledge Falmer.

Featherstone, S. (2004) Smooth Transitions. Nursery World, 104 (3919), 14-15. 
A. CASTRO ZUBIZARRETA, P. EZQUERRA MUÑOZ Y J. ARGOS GONZÁLEZ

PROFUNDIZANDO EN LA TRANSICIÓN ENTRE EDUCACIÓN INFANTIL Y EDUCACIÓN PRIMARIA...

Gairín, J. (2005) El reto de la transición entre etapas educativas. Aula de Innovación Educativa, 142, 12-17.

Hendy, L. y WhiteBreAD, D. (2000) Interpretations of independent learning in the Early Years. International Journal of Early Years Education, 8 (3), 245-52.

Janus, M. (2011) Transition to School, en Lavericky, M. D. y RenCK, M. (eds.) Transitions to Early Care and Education. Indiana, Springer, 177-187.

Janus, M.; Lefort, J.; CAMEROn, R. y KopeChanski, L. (2007) Starting kindergarten: What do we know about issues affecting the transition to school for children with special needs? Canadian Journal of Education, 30, 628-648.

Kaplun, C.; DocketT, S. y Perry, B. (2017) The starting school study: Mothers' perspectives of transition to school. Australasian Journal of Early Childhood, 42 (4), 56-66.

KINKEAD-ClARK, Z. (2015) Ready for big school: making the transition to primary school - a Jamaican perspective. International Journal of Early Years Education, 23 (1), 67-82.

KinKEAD-Clark, Z. (2016) Select Caribbean teachers' perspectives on the socio-emotional skills children need to successfully transition to primary school. Early Child Development and Care, 187 (9), 1403-1412.

Lara-Cinisomo, S.; Fuligni, A. S.; Ritchie, S.; Howes, C. y Karoly, L. (2008) Getting ready for school: An examination of early childhood educators' belief systems. Early Childhood Education Journal, 35, 343-349.

Pianta, R. y Kraft-SAYre, M. (2003) Successful kindergarten transition. Baltimore, Brookes, MD.

Rimm-Kaufman, S. E. y Pianta, R. C. (2000) An ecological perspective on the transition to kindergarten: A theoretical framework to guide empirical research. Journal of Applied Developmental Psychology, 21, 491-511.

Rosier, K. y McDonald, M. (2011) Promoting positive education and care transitions for children. Communities and Families Clearing house Resource Sheet. Consultado el 28 de diciembre de 2013. http://www.aifs.gov.au/cafca/pubs/sheets/rs/rs5.html.

Sakellariou, M. y Sivropoulou, I. (2010) Family and Kindergarten Cooperation within the Framework of Children's Transition from Kindergarten to Primary School. International Journal of Interdisciplinary Social Sciences, 5 (4), 345-360.

Sayers, M.; West, S.; Lorains, J.; Laidlaw, B.; Moore, T. G. y Robinson, R. (2012) Starting school: A pivotal life transition for children and their families. Family Matters, 90, 45-56.

Schulting, A. B.; Malone, P. S. y Dodge, K. A. (2005) The effect of school-based kindergarten transition policies and practices on child academic outcomes. Development and Psychopathology, 41 (6), 860-871.

UnICEF (2009) Child Friendly Schools Manual. New York, United Nations Children's Fund.

Vogler, P.; Crivello, G. y WoOdHEAD, M. (2008) La investigación sobre las transiciones en la primera infancia: Análisis de nociones, teorías y prácticas. Cuadernos sobre Desarrollo Infantil Temprano, 48. La Haya, Fundación Bernard van Leer.

Yi HunG LAU, E. (2014) Chinese parents' perceptions and practices of parental involvement during school transition. Early Child Development and Care, 184 (3), 403-415. 